\title{
Ion and Chain Mobility in a Tetrazole Proton-Conducting Polymer
}

\author{
R. Casalini,* B. L. Chaloux, C. M. Roland, and H. L. Ricks-Laskoski \\ Naval Research Laboratory, Chemistry Division, Code 6120, Washington, D.C. 20375-5342, United States
}

Supporting Information

ABSTRACT: The morphology, relaxation properties, and conductivity of the statistical copolymer polystyrenic (alkoxy $1 H$-tetrazole-co-alkoxy nitrile), an anhydrous proton conductor, were measured. The material phase-separates into hard and soft domains, the latter corresponding to a phase richer in the pendant tetrazole groups. Using dielectric and mechanical spectroscopies, two relaxation processes were observed, the slower associated with local segmental dynamics of the backbone and the higher-frequency process involving motion of the tetrazole moieties. The latter is coupled to the ionic conductivity, which means that below the principal glass transition of the material $(\sim 313 \mathrm{~K})$ the conductive mechanism remains active. Thus, the usual compromise in proton exchange membranes between mechanical stability and ion conductivity can be avoided.

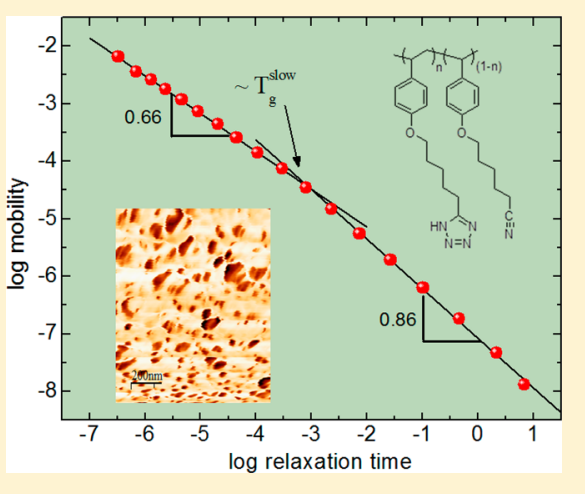

\section{INTRODUCTION}

The attraction of new energy sources has stimulated development of both new and existing technologies. An obvious enabling factor for devices such as photovoltaic and fuel cells is the combination of high performance and low cost. Polymeric materials are especially promising given, along with their inherent processability, the multitude of chemical structures available to tailor properties. For fuel cells, proton exchange membranes (PEMs) with high proton conductivity but very small electron conductivity are required. The current state-ofthe-art PEMs are made with Nafion, a perfluorinated polymer. However, the proton conductivity of Nafion is strongly dependent on its water content. This limits the operating temperature to below $373 \mathrm{~K}$ and requires precise control of hydration levels to obtain optimal cell performance. A better PEM would have the proton conductivity of hydrated Nafion without requiring water molecules for the conduction mechanism.

To produce materials possessing the desired conducting properties, the synthetic process must be guided by an understanding of the physical properties. In this work we describe dielectric and mechanical measurements on a protonconducting polymer, the statistical copolymer polystyrenic (alkoxy $1 H$-tetrazole-co-alkoxy nitrile) (PS-(Tz-co-CN)), the synthesis of which is described elsewhere. ${ }^{1}$ In anhydrous conditions PS-(Tz-co-CN) has a DC conductivity $\sigma_{\mathrm{DC}}=4 \times$ $10^{-12} \mathrm{~S} / \mathrm{cm}$ at ambient temperature and $2 \times 10^{-6} \mathrm{~S} / \mathrm{cm}$ at 388 $\mathrm{K}$, which is comparable to undoped imidazole and triazolecontaining polymers ${ }^{2,3}$ and higher than that of anhydrous Nafion (a sulfonic acid polymer). ${ }^{4}$ However, this conductivity is well below that of hydrated Nafion $\left(10^{-2} \mathrm{~S} / \mathrm{cm}\right.$ at $\left.353 \mathrm{~K}\right)$, small-molecule-doped materials including organic acid- and base-impregnated polybenzimidazole membranes, ${ }^{5}$ strong aciddoped aromatic or heterocyclic polymers, ${ }^{6}$ doped polymers structurally similar to PS-(Tz-co-CN) (e.g., imidazoles, triazoles, and tetrazoles), ${ }^{2}$ and doped tetrazole-containing polymers, with $\sigma_{\mathrm{DC}}$ as high as $10^{-3} \mathrm{~S} / \mathrm{cm}$ at high $T^{7,8}$ As $\sigma_{\mathrm{DC}}$ of PS-(Tz-co-CN) is comparable to that of other undoped azole-based materials, ${ }^{2,3}$ we expect that its conductivity can be similarly improved several orders of magnitude by doping. Thus, although in the anhydrous and undoped state the conductivity of PS-(Tz-co-CN) is too low for practical PEM applications, this work focuses on understanding the physical properties of the material to guide future chemical modifications that can further improve the DC conductivity to yield viable PEM and related devices since high DC conductivity is not the only required property.

\section{EXPERIMENTAL SECTION}

The synthesis of the PS-(Tz-co-CN) (Figure 1) is described in detail elsewhere. ${ }^{1}$ The relative amount of the two monomers, $n$

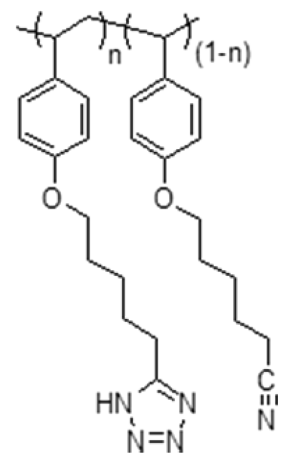

Figure 1. Chemical structure of PS-(Tz-co-CN), $n=0.90$.

Received: January 7, 2014

Revised: March 7, 2014

Published: March 25, 2014 
$=0.90$, was measured using proton nuclear magnetic resonance spectroscopy ( ${ }^{1} \mathrm{H}$ NMR $) .{ }^{1}$ On the basis of the molecular weight $(11000 \mathrm{D})$ of the precursor poly(4-vinylphenol), we assume $M_{\mathrm{w}}=23300 \mathrm{D}$ for the PS-(Tz-co-CN).

Dielectric spectra were measured using a Novocontrol Alpha impedance analyzer. The sample cell consisted of stainless steel parallel plates (diameter $=16 \mathrm{~mm}$ ) with $0.1 \mathrm{~mm}$ Teflon spacers; the cell was assembled by pressing the material between the plates at $\sim 388 \mathrm{~K}$. The stainless steel electrodes are impervious to ions from the PS- $(\mathrm{Tz}-\mathrm{co}-\mathrm{CN})$ and thus function as blocking electrodes. Vacuum was maintained during the dielectric measurements, with temperature controlled by a custom closed-cycle helium cryostat (Cryo Ind.). An initial series of measurements at constant $T=383 \mathrm{~K}$ over a period of $64 \mathrm{~h}$ showed no change in the dielectric response of the material. Mechanical measurements employed an Anton Parr MCR 502 rheometer (parallel plate geometry: diameter $=8$ $\mathrm{mm}$; typical thickness $=1.2 \mathrm{~mm}$ ). The sample was also molded at ca. $383 \mathrm{~K}$ in vacuo and kept under a nitrogen atmosphere during the mechanical measurements.

Atomic force microscopy (AFM) in tapping mode was done using an Asylum Cypher ES AFM, with a nitrogen atmosphere at $301 \mathrm{~K}$. Samples (thickness $\sim 0.8 \mathrm{~mm}$ ) were cast on a Teflon substrate from $21 \%$ by weight dimethyl sulfoxide solution dried at $373 \mathrm{~K}$ under nitrogen. Differential scanning calorimetry (DSC) was carried out with a TA Q100 at a heating rate of 10 $\mathrm{K} \mathrm{min}^{-1}$ and a helium purge. Changes in the sample morphology are possible during high-temperature annealing; therefore, to maintain a consistent morphology among the different measurements, all experiments and sample preparation were conducted at temperatures not exceeding $388 \mathrm{~K}$.

\section{RESULTS}

Dielectric Spectroscopy. Dielectric spectra of PS-(Tz-co$\mathrm{CN}$ ) at different temperatures (Figure 2) indicate the presence

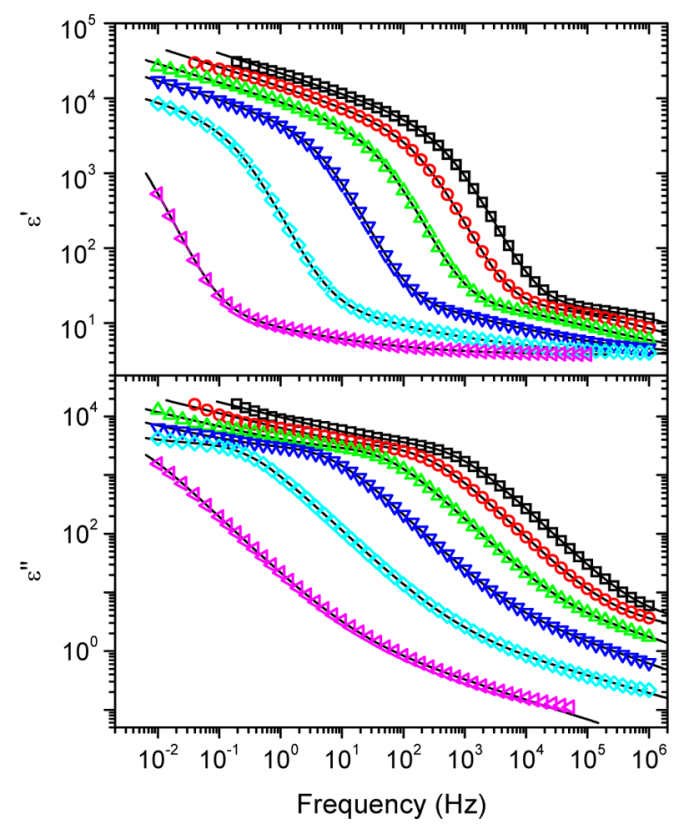

Figure 2. Real (upper) and imaginary (lower) parts of the dielectric permittivity of PS-(Tz-co-CN) at temperatures (left to right) $T(\mathrm{~K})=$ $302.2,319.4,336.2,353.4,370.5$, and 387.7. The solid lines are the fits of eq 1 . of three processes: (i) electrode polarization (EP), (ii) the $\alpha$ (or structural) relaxation, and (iii) a secondary $\beta$ relaxation. EP is due to the accumulation of free charges at the surface of the blocking electrodes, manifested as large increases with decreasing frequency in both the real $\left(\varepsilon^{\prime}\right)$ and imaginary $\left(\varepsilon^{\prime \prime}\right)$ components of the permittivity. The permittivity becomes essentially constant at low frequency (Figure 2), at a value of $\varepsilon^{\prime}$ $\sim 10^{5}$ that is about 3 orders of magnitude larger than the permittivity of any material having only a permanent molecular dipole. This confirms the underlying process as EP rather than molecular reorientation. The $\alpha$-relaxation, involving reorientation of permanent dipoles on the polymer backbone, is largely obscured by the large EP at lower temperatures, with the dispersion beginning to emerge at temperatures above about $323 \mathrm{~K}$. The $\beta$ relaxation is only evident in the glassy state at low temperatures, where the contribution of the other processes is negligible. This secondary process, involving intramolecular degrees of freedom, is due to motion of pendant groups (e.g., the polar tetrazole moiety).

We assume the three processes are independent and additive, so that the complex permittivity can be described by the sum of the three contributions, along with a frequency-independent constant, $\varepsilon_{\infty}$

$$
\varepsilon^{*}(f)=\varepsilon_{\mathrm{EP}}^{*}(f)+\varepsilon_{\alpha}^{*}(f)+\varepsilon_{\beta}^{*}(f)+\varepsilon_{\infty}
$$

We simultaneously fit eq 1 to both the real and imaginary parts of $\varepsilon^{*}(f)$; the calculated curves are included in Figure 2 .

To describe the EP contribution we used the empirical modification of the MacDonald model $^{9}$ proposed by Fragiadakis et al. ${ }^{10}$

$$
\varepsilon_{\mathrm{EP}}^{*}(f)=\frac{\Delta \varepsilon_{\mathrm{EP}}}{\left(i 2 \pi f \tau_{\mathrm{EP}}\right)^{1-n}+i 2 \pi f \tau_{\mathrm{EP}}}
$$

where $\Delta \varepsilon_{\mathrm{EP}}$ and $\tau_{\mathrm{EP}}$ are the apparent dielectric strength and relaxation time, respectively, and $n(0<n \leq 1)$ is related to the roughness of the electrode surface. ${ }^{11}$ For a single charge carrier with charge $q, \Delta \varepsilon_{\mathrm{EP}}$ and $\tau_{\mathrm{EP}}$ are related to the charge mobility, $\mu$, and concentration, $p$, as ${ }^{9}$

$$
\begin{aligned}
& \tau_{\mathrm{EP}}=\frac{L}{2 L_{\mathrm{D}}} \frac{\varepsilon_{0} \varepsilon_{\mathrm{s}}}{q \mu p} \\
& \Delta \varepsilon_{\mathrm{EP}}=\left(\frac{L}{2 L_{\mathrm{D}}}-1\right) \varepsilon_{\mathrm{s}}
\end{aligned}
$$

where $\varepsilon_{0}$ is the vacuum permittivity; $\varepsilon_{\mathrm{s}}$ is the static dielectric constant; and $L$ is the electrode spacing. The Debye length $L_{\mathrm{D}}$ is

$$
L_{\mathrm{D}}=\left(\frac{\varepsilon_{0} \varepsilon_{\mathrm{s}} k T}{q^{2} p}\right)^{1 / 2}
$$

where $k$ is the Boltzmann constant. Since $\Delta \varepsilon_{\mathrm{EP}}$ and $\tau_{\mathrm{EP}}$ have different dependences on $\mu$ and $p$, the values of $\mu$ and $p$ can be extracted from the measured $\varepsilon_{\mathrm{EP}}^{*}$. 10

For the dipolar loss peaks the Kohlraush-Williams-Watts function (KWW) was used to describe the $\alpha$-relaxation and the Havriliak-Negami function (HN) used for the $\beta$-process ${ }^{12}$ 


$$
\begin{aligned}
\varepsilon_{\alpha}^{*}(f)+\varepsilon_{\beta}^{*}(f)= & \Delta \varepsilon_{\alpha} L_{i \omega}\left[-\frac{d \varphi_{\alpha}\left(t, \tau_{\alpha}\right)}{d t}\right] \\
& +\frac{\Delta \varepsilon_{\beta}}{\left[1+\left(i 2 \pi f \tau_{\beta}\right)^{a}\right]^{b}}
\end{aligned}
$$

with

$$
\varphi_{\alpha}\left(t, \tau_{\alpha}\right)=\exp \left[-\left(t / \tau_{\alpha}\right)^{\beta_{\mathrm{KWW}}}\right]
$$

$L_{i \omega}$ indicates the Laplace transform; $\tau_{i}$ are the relaxation times; $\Delta \varepsilon_{i}$ are the dielectric strengths; and $\beta_{\mathrm{KWw}}\left(0<\beta_{\mathrm{KWw}} \leq 1\right)$ and $a$ and $b(0<a, b \leq 1)$ are the shape parameters for the KWW and $\mathrm{HN}$ functions, respectively. Representative examples of the fit functions and their superposition are shown in Figures 3 and 4.

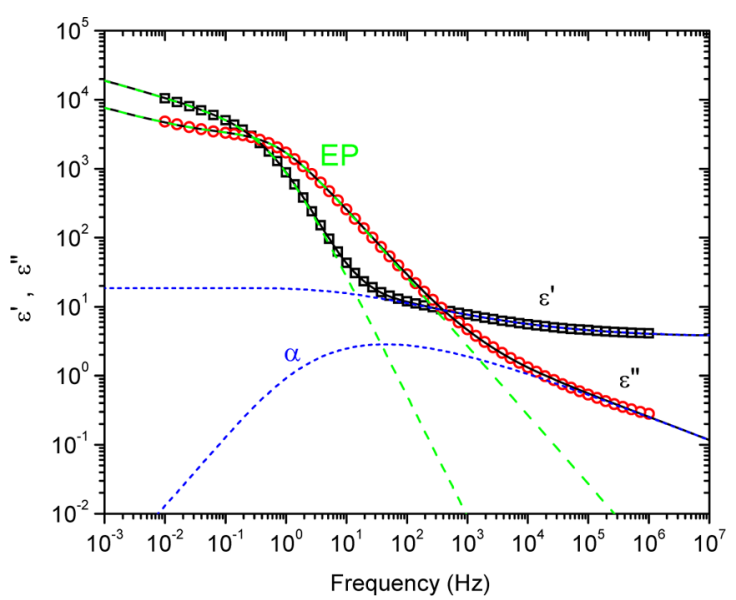

Figure 3. Real (squares) and imaginary (circles) components of the permittivity at $T=323.7 \mathrm{~K}$. The solid lines are the fits of eq 1 ; the KWW function $\left(\beta_{\mathrm{KWW}}=0.34 ; \log \left(\tau_{\alpha}\right)=-2.6\right)$ and the electric polarization $\left(\log \left(\tau_{\mathrm{EP}}\right)=-0.59 ; n=0.76\right)$ are represented by short and long dashes, respectively.

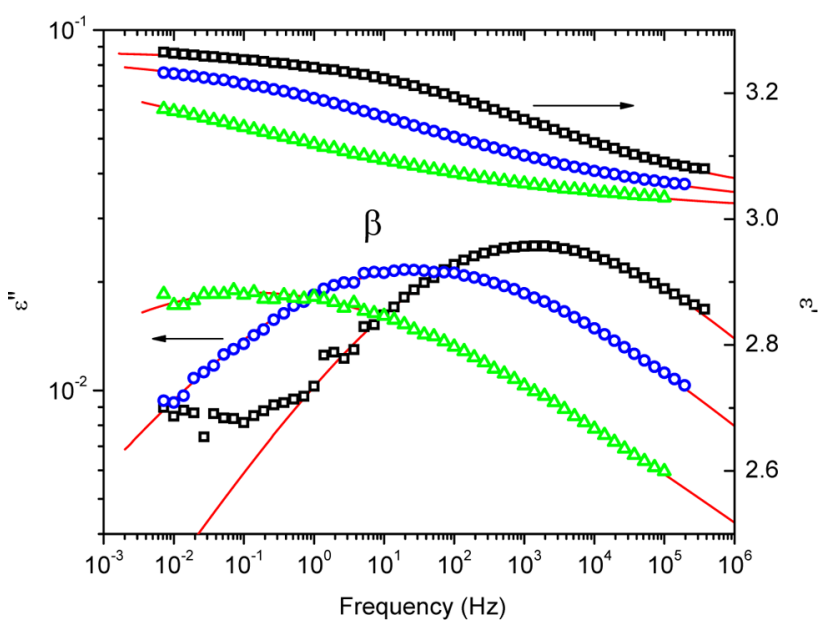

Figure 4. Real and imaginary part of the permittivity at (left to right) $T(\mathrm{~K})=117.1,137.0$, and 157.1. The solid lines are the fitted HN relaxation function.

The relaxation times $\tau_{\alpha}$ and $\tau_{\mathrm{EP}}$ showed non-Arrhenius temperature dependences, with an apparent activation energy that increased with decreasing temperature. These temperature dependences can be described by the Vogel-FulcherTammann (VFT) equation

$$
\tau=\tau_{0} \exp \left(\frac{B}{T-T_{0}}\right)
$$

where $\tau_{0}, T_{0}$, and $B$ are constants; ${ }^{12}$ the obtained values for the VFT parameters are listed in Table 1 . The $\beta$ process exhibits

Table 1. VFT (Equation 8) Fit Parameters for the Relaxation Times $^{a}$

$\begin{array}{lcccc} & \log \left(\tau_{0}[\mathrm{~s}]\right) & B[\mathrm{~K}] & T_{0}[\mathrm{~K}] & T(\tau=100 \mathrm{~s})[\mathrm{K}] \\ \tau_{\mathrm{EP}} & -8.0 \pm 0.2 & 1530 \pm 80 & 234 \pm 3 & 300 \pm 3 \\ \tau_{\alpha} & -14.3 \pm 0.4 & 2700 \pm 200 & 221 \pm 4 & 292 \pm 6 \\ \tau_{\mathrm{M}}^{\text {slow }} & -11.4 \pm 0.7 & 1400 \pm 200 & 274 \pm 4 & 320 \pm 6 \\ \tau_{\mathrm{M}}^{\text {fast }} & -14.3 \pm 0.4 & 2700 \pm 200 & 211 \pm 4 & 282 \pm 6\end{array}$

${ }^{a}$ For $\tau_{\mathrm{M}}^{\text {fast }}$ the data were shifted in temperature by $10.5 \mathrm{~K}$ to superimpose with $\tau_{\alpha}$.

Arrhenius behavior, $\tau_{\beta}=\tau_{\beta}^{\infty} \exp \left(E_{\beta} / R T\right)$ with $\log \left(\tau_{\beta}^{\infty}[s]\right)=$ $-15.2 \pm 0.2$ and $E_{\beta}=33.8 \pm 0.3 \mathrm{~kJ} / \mathrm{mol}$ (Figure 5). This

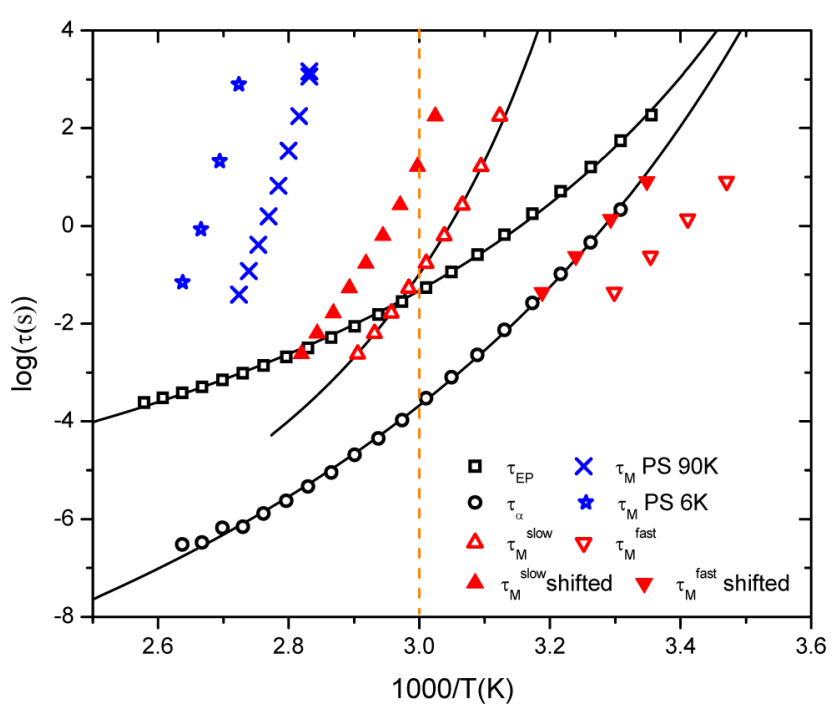

Figure 5. Arrhenius plot of the relaxation times from dielectric (squares and circles) and mechanical (normal and inverted triangles) measurements on PS-(Tz-co-CN). The solid inverted triangles are the $\tau_{\mathrm{M}}^{\text {fast }}$ shifted by $10.5 \mathrm{~K}$, yielding superpositioning with the dielectric $\tau_{\alpha}$. Included for comparison are the mechanical relaxation times for polystyrenes having molecular weights bracketing that of the polymer herein. ${ }^{15}$ The solid lines represent eq 8 . The vertical dotted line denotes the temperature (333 K) at which $\tau_{\alpha}$ and $\mu$ decouple.

activation energy is about half the value of $24 R T_{\mathrm{g}}$ expected for a Johari-Goldstein-type secondary relaxation; ${ }^{13,14}$ thus, it is identified as a local motion of the pendant groups. The $\beta$ process could be related to the fast motion of the $\mathrm{CN}$ side groups, but more work is necessary to confirm this possibility.

Mechanical Relaxation. The modulus measured at a fixed frequency of $1 \mathrm{~Hz}$ is shown in Figure 6 as a function of temperature. Two processes are evident, with respective peaks at $T \cong 303$ and $338 \mathrm{~K}$. The molecular weight of the PS-(Tz-co$\mathrm{CN})$ backbone is less than the value $\left(=18100 \mathrm{D}^{16}\right)$ for entanglement interactions of polystyrene (PS), so the highertemperature process cannot be due to terminal relaxation. Both peaks are attributed to local segmental dynamics. 


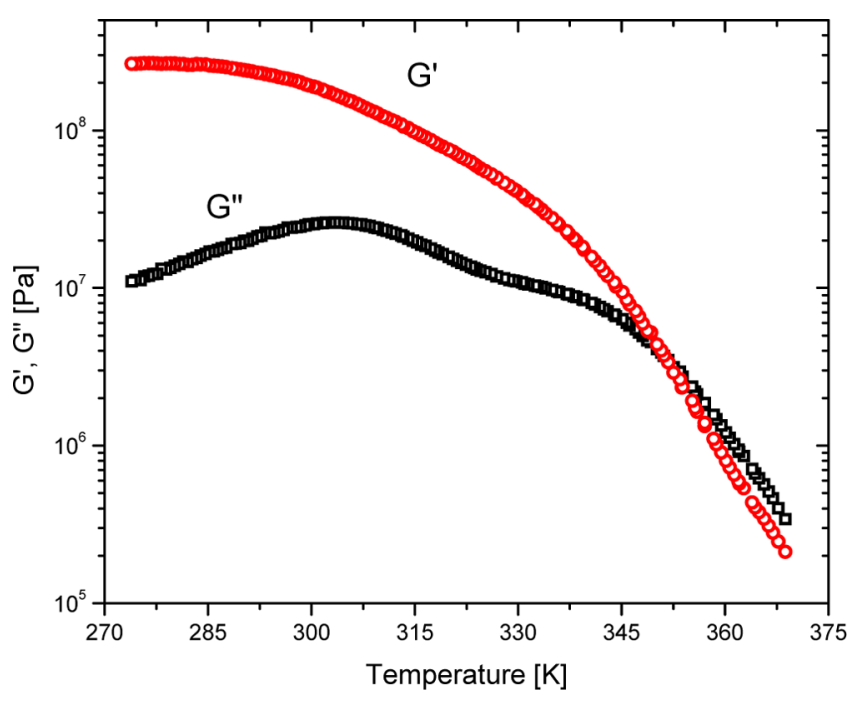

Figure 6. Storage (circles) and loss (squares) moduli of PS-(Tz-co$\mathrm{CN}$ ) measured at $1 \mathrm{~Hz}$ during cooling at $0.1 \mathrm{~K} / \mathrm{min}$.

To understand the origin of two processes, we made isothermal measurements of the mechanical modulus at low temperatures (Figure 7). Equation 7 was fit to the higher-

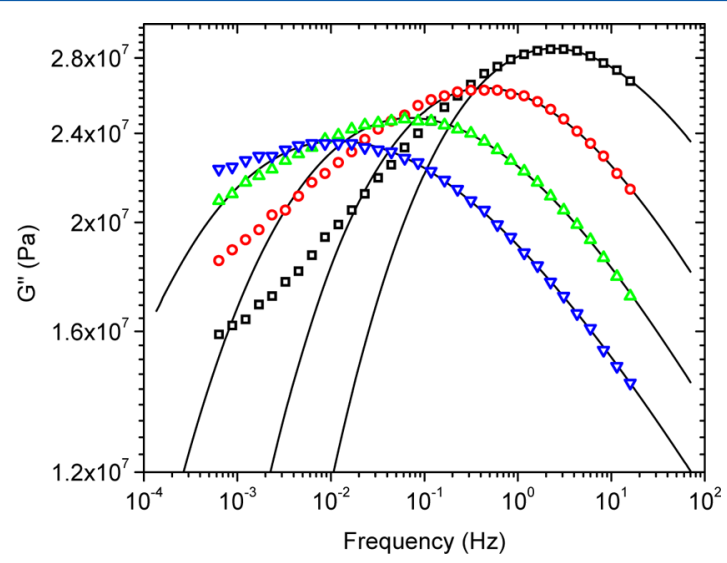

Figure 7. Isothermal spectra of the loss modulus of PS-(Tz-co-CN) at (left to right): $T(\mathrm{~K})=288.1,293.1,298.1$, and 333.1. The solid lines are fits of the KWW function to the higher-frequency peak.

frequency peak to determine $\tau_{\mathrm{M}}^{\mathrm{fast}}$. The exponent $\beta_{\mathrm{KWW}} \cong 0.2$ indicates a somewhat broader peak than the dielectric $\alpha$ relaxation peak, for which $\beta_{\mathrm{KWw}} \sim 0.3$. Because only part of the lower-frequency peak was present in most spectra, we determined the relaxation time at $T=329 \mathrm{~K}$ by fitting eq 7 to the spectrum and then calculated $\tau_{\mathrm{M}}^{\text {slow }}$ at the other temperatures using frequency-temperature shift factors that superpose the spectra toward lower frequencies (see Supporting Information for the master curve). The obtained mechanical relaxation times are displayed in Figure 5, along with the relaxation times from the dielectric spectra. Invariably segmental relaxation times are longer when determined by mechanical measurements than the values extracted from dielectric spectra. ${ }^{12,17-20}$ The origin of this difference is a current topic of investigation. ${ }^{21,22}$ The faster mechanical relaxation peak appears to correspond to the dielectric $\alpha$ relaxation, the mechanical $\tau_{\mathrm{M}}^{\text {fast }}$ superpose on the dielectric $\tau_{\alpha}$, by a 10.5 degrees shift. On the other hand, the $\tau_{\mathrm{M}}^{\text {slow }}$ are longer than the relaxation times of any of the dielectric processes; the corresponding dielectric peak would fall at frequencies for which they would be hidden by the large ionic conductivity and consequent EP. The $\tau_{\mathrm{M}}^{\text {slow }}$ exhibit VFT behavior, with the parameters for eq 8 listed in Table 1 . If the slow relaxation is associated with a distinct phase of the material, the corresponding glass transition temperature $T_{\mathrm{g}}^{\text {slow }}=320 \mathrm{~K}$ (or $330 \mathrm{~K}$ applying the same temperature shift as for $\tau_{\mathrm{M}}^{\text {fast}}$; solid symbols in Figure 5). Therefore, the observed dielectric $\alpha$ relaxation is associated with the fast mechanical relaxation (softer phase of PS-(Tet-co-CN)), while the slow relaxation (hard phase) is observed only by mechanical spectroscopy due to electrode polarization affecting the dielectric measurements.

An interesting aspect of the rheological measurements is that even at temperatures well above $T_{\mathrm{g}}$ viscous flow is suppressed. The dynamic mechanic data are suggestive of solid-like behavior: the modulus varies sublinearly with frequency, having an exponent that decreases at lower frequencies (see Supporting Information). This suppression of flow can be ascribed to the effect of hydrogen bonding between the tetrazole pendent groups, which gives rise to a quasi-network. This association of the polymer chains has potential utility in applications such as PEM, by enabling stable membranes to be obtained without the need for chemical cross-linking.

Differential Scanning Calorimetry. DSC measurements (Figure 8, lower panel) reveal a broad transition around room

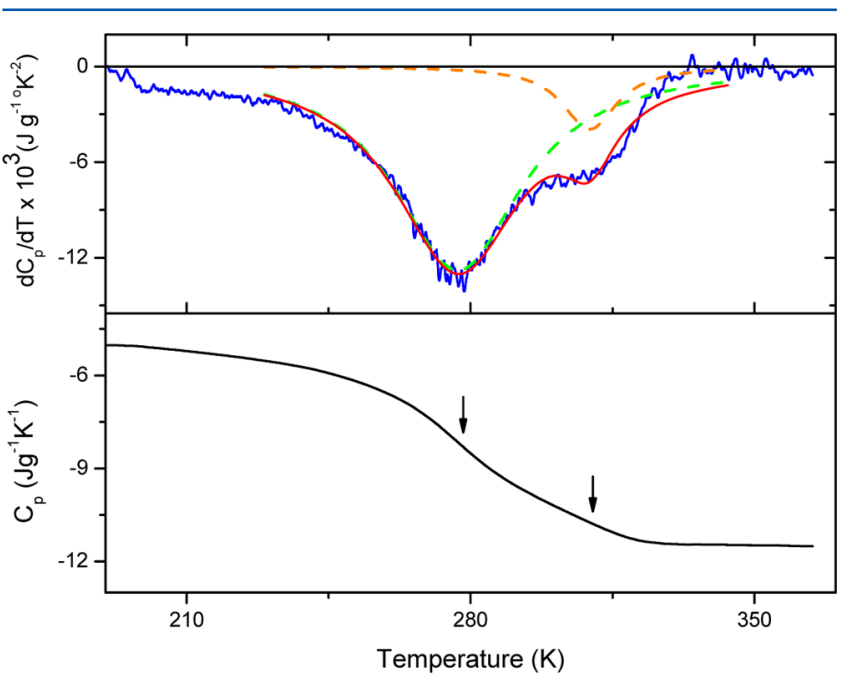

Figure 8. (Lower panel) Specific heat of PS-(Tz-co-CN) measured during heating. (Upper panel) Temperature derivative; the solid line is the fit assuming two Lorentzian functions and serves to guide the eye. The peak temperatures are indicated by arrows in the lower panel.

temperature; there is no indication of other processes, including crystallization. The observed transition is actually the superposition of two processes, as seen by plotting the temperature derivative of the heat capacity (upper panel of Figure 8). Fitting two curves to these peaks, we estimate the transition temperatures to be $276.9 \pm 0.1 \mathrm{~K}$ and $309.5 \pm 0.4 \mathrm{~K}$. These values are close to the $T_{\mathrm{g}}$ 's found from the mechanical measurements and consistent with a phase-segregated morphology.

Atomic Force Microscopy. To investigate the phase segregation revealed by the mechanical and DSC measurements, tapping mode AFM was employed. Since the AFM measurements of the surface of the film may not be representative of the bulk, we prepared a thicker film $(\sim 0.8$ 

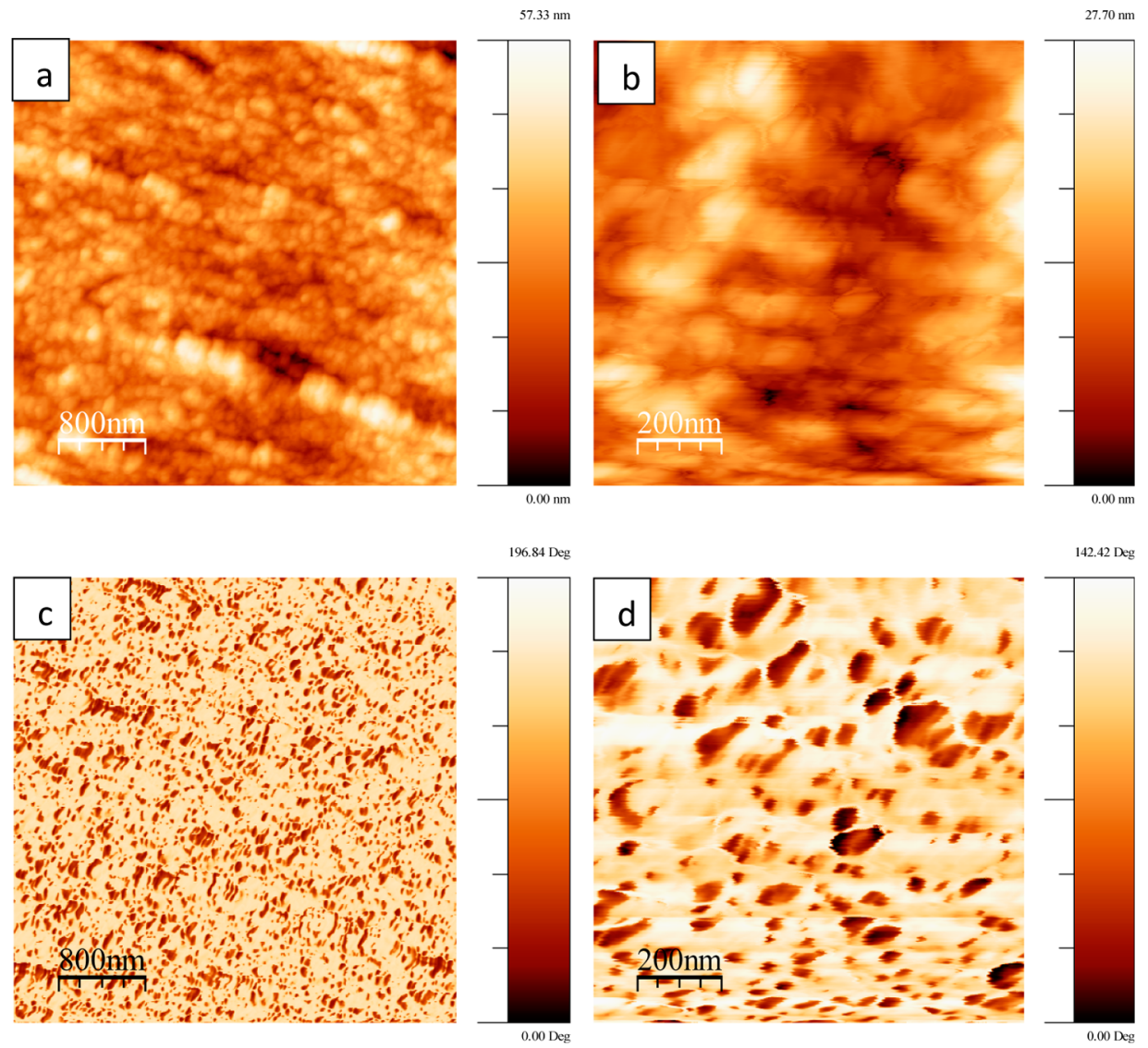

Figure 9. AFM images of morphology (upper panels) and the corresponding phase (lower panels measured in tapping mode at $T=301 \mathrm{~K}$ ) for a cross-section of PS-(TZ-co-CN). Darker areas in the phase images correspond to more compliant domains.

$\mathrm{mm}$ ) and measured its cross-section after cutting it with a sharp blade. The morphological images of the cross section of a PS(Tz-co-CN) film (panels a and b in Figure 9) show a quite smooth surface (average height $\sim 28 \mathrm{~nm}$ with a roughness $\sim 7$ $\mathrm{nm}$ over an area of $4 \times 4 \mu \mathrm{m}$ ). AFM phase images (panels $\mathrm{c}$ and $d$ in Figure 9) reveal two phases defined by their different stiffness. The concentrations of the domains are roughly equal, with a broad distribution of dimensions with maximum dimensions of about $100 \mathrm{~nm}$. The presence of two domains is consistent with the mechanical and DSC data since the temperature of the AFM measurements $(301 \mathrm{~K})$ is intermediate between the transition temperatures (see Figure 5 and Figure 8 ). Thus, the hard and soft domains correspond to respective slow and fast dynamic processes.

SAXS measurements (not shown) of the PS- $(\mathrm{Tz}-\mathrm{co}-\mathrm{CN})$ give no indication of any structure, consistent with the extremely disordered morphology observed by AFM, as well as possibly small differences in electron density between the two phases.

\section{DISCUSSION}

The slower relaxation process seen in the mechanical measurements is identified with the segmental dynamics of the polymer backbone, consistent with data for a PS homopolymer (Figure 5), ${ }^{15}$ although there is internal plasticization in the present material by the pendant tetrazole moieties. The faster mechanical relaxation and the corresponding dielectric $\alpha$-relaxation are ascribed to motion of the side groups, which is faster (being less intramolecularly cooperative) and involves the more polar part of the chain. This side group motion should be coupled to the ionic conductivity since it is the tetrazole group that participates in the conduction process. ${ }^{23}$ The mechanism is by hopping of protons between sites over an extended hydrogen bond network (Grotthuss mechanism $^{24}$ ) formed by the tetrazole groups. Flexibility of the side chain, ease of rotation, and proximity of the tetrazole rings are therefore important factors governing proton mobility. The AFM images and the mechanical measurements both indicate a phase-separated morphology, with the softer phase associated with the faster process. Attribution of the two phases from a molecular point of view is not straightforward. The soft domains are almost ten times larger than what is expected from the length of the side chain; therefore, the structure must be rather complex, arising from the very different nature of the pendant group and the backbone. Certainly, we expect the softer phases to be enriched in the more mobile tetrazole, although molecular dynamic simulations would be required to better quantify the morphology. Conceivably, different local ordering of the pendant groups, without substantial change in pendant groups concentration, could underlie the observed difference in mechanical stiffness.

Analyzing the EP using eqs 3-5, we obtain the temperature dependences of the charge density and mobility (Figure 10). The latter exhibits VFT-like behavior (eq 8), with $T_{0}=242 \pm 3$ $\mathrm{K}$ and $B=1270 \pm 70 \mathrm{~K}$. To assess the coupling between ion mobility and the segmental dynamics, Figure 10 is a plot of $\mu$ versus $\tau_{\alpha}$. The data follow the empirical Debye-StokesEinstein fractional relation ${ }^{25}$

$$
\mu \propto \tau_{\alpha}^{m}
$$




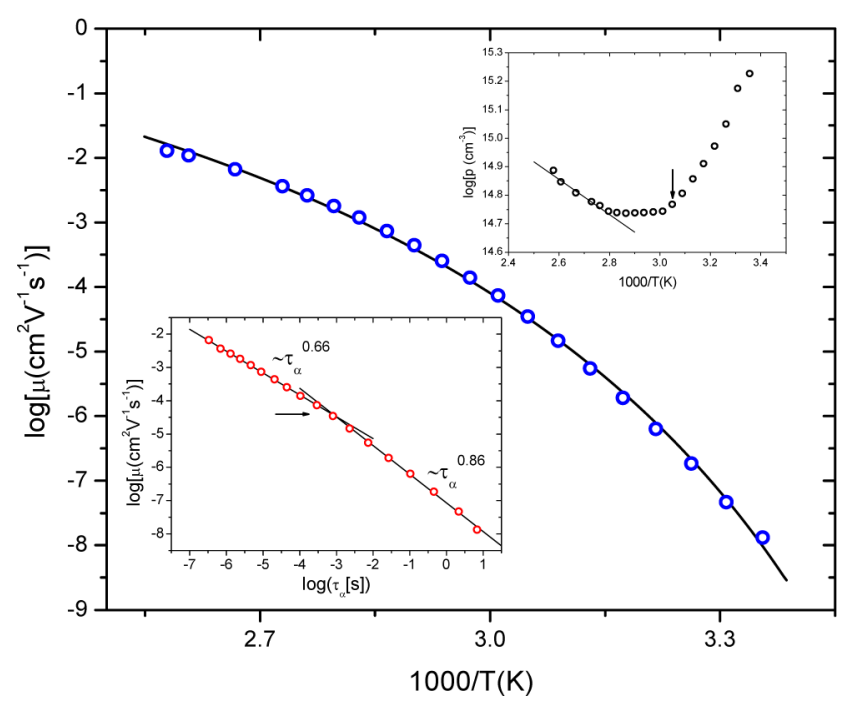

Figure 10. Temperature dependence of the ion mobility calculated from the EP analysis. The solid line is the VFT fit. The lower inset is a double logarithmic plot of the mobility versus dielectric relaxation time, with the lines representing linear fits that reveal two regions having different coupling between $\mu$ and $\tau_{\alpha}$; the arrow indicates the location of this crossover in behavior. The upper inset shows the temperature dependence of the charge density, with Arrhenius behavior denoted at higher $T$. The arrow corresponds to the temperature having the mobility indicated by an arrow in the lower inset.

There is a change from a larger exponent at low temperature $(m=0.86)$ to a smaller value at higher temperatures $(m=$ $0.66)$, the decrease indicating less coupling with increasing temperature. It is interesting to note that this loss of coupling (denoted by arrows in Figure 10) becomes manifest around $T$ $=333 \mathrm{~K}$, which is just above the glass transition temperature for the slow segmental (backbone) process (see Figure 5 and Figure 8). Reflecting different proton transport mechanisms, the temperature dependence of the proton mobility in PS- $(\mathrm{Tz}-$ $c o-\mathrm{CN})$ is quite different from that for Nafion. For the latter, proton transfer relies on proton mobility in water. For Nafion at room temperature, $\mu \sim 10^{-4} \mathrm{~S} / \mathrm{cm},{ }^{26}$ which is more than 3 orders of magnitude higher than the value observed herein for PS-(Tz-co-CN) at the same T. At higher temperatures Nafion dehydrates, and the mobility increase is only an order of magnitude at $368 \mathrm{~K}$. In contrast, for PS-(Tz-co-CN), in which proton mobility is governed by the pendant group mobility, the temperature dependence is stronger. Over the range from 298 to $368 \mathrm{~K}$, the relaxation time for the pendant group decreases 6 orders of magnitude, reaching a value at $368 \mathrm{~K}$ of ca. $10^{-6} \mathrm{~s}$ (Figure 5), i.e., commensurate with the relaxation time of a liquid. Although the proton mobility in pure tetrazole is unknown, for the related $\mathrm{N}$-heterocyclic imidazole, a lower limit of $\mu=4 \times 10^{-3} \mathrm{~cm}^{2} \mathrm{~V}^{-1} \mathrm{~s}^{-1}$ at $368 \mathrm{~K}$ has been reported, ${ }^{27}$ close to that found herein for PS-(Tz-co-CN).

The temperature dependence of the charge density also shows the presence of two asymptotic regimes at high and low temperatures, with a crossover region in which the ion concentration is almost constant (Figure 10 inset). Below $T$ $\sim 333 \mathrm{~K}, p$ increases. At high temperatures (>353 K) the ion concentration exhibits properties typical of homogeneous ion conductors: ${ }^{10}$ Arrhenius behavior, $p=p_{\infty} \exp \left(-E_{\text {act }} / R T\right)$ with $E_{\mathrm{a}}=11.9 \pm 0.9 \mathrm{~kJ} / \mathrm{mol}$ and $p_{\infty}=(3 \pm 1) \times 10^{16} \mathrm{~cm}^{-3}$. Assuming full dissociation of all protons and from the stoichiometry of the PS-(Tz-co-CN), we expect a maximum value of $p=1.2 \times 10^{21} \mathrm{~cm}^{-3}$; therefore, although the ion conductivity is relatively high, the proton dissociation is very limited. Note that this proton dissociation is only an estimate since the modified Macdonald model is only accurate to an order of magnitude. ${ }^{10}$ On the basis of the aqueous dissociation constants of $1 \mathrm{H}$-tetrazole, ${ }^{28}$ the dissociation energy of $1 \mathrm{H}$ tetrazole is estimated to be $126 \mathrm{~kJ} / \mathrm{mol}$ assuming a second tetrazole is the proton acceptor or $28 \mathrm{~kJ} / \mathrm{mol}$ if water is the acceptor. The latter is unlikely since the samples were extensively dried prior to our measurements. Thus, the activation energy of $p$ is significantly smaller than the dissociation energy.

The anomalous increase in the charge density at low temperatures possibly results from an apparent increase of $\Delta \varepsilon_{\mathrm{EP}}$ due to other polarization processes, for example, charge accumulation at interfaces within the sample (Sillars polarization); however, no other process is evident in the dielectric spectra. The observed behavior could be related to the phasesegregated nature of the material. The majority of the charge transport occurs through the (soft) tetrazole-rich phases, whereby any diffusion of ions into the (hard) PS-rich phase corresponds to a loss of ions. For temperature below the glass transition of the PS-rich phase (ca. $313 \mathrm{~K}$ ), diffusion of ionic species into it is suppressed; this suppression of ion depletion manifests as an apparent increase in the number of ionic species. On the other hand, at high temperature a "loss" of ions from the fast to the slow phase would cause a smaller activation energy of $p$. This interpretation is consistent with the increased coupling between the segmental relaxation of the tetrazole-rich phase with the ion mobility since ions that migrate into the slow phase would be less correlated with the faster relaxation.

\section{CONCLUSIONS}

An investigation of PS-(Tz-co-CN) ion transport properties using dielectric and mechanical spectroscopies, DSC, and AFM shows that PS-(Tz-co-CN) forms a two-phase system, with soft domains richer in tetrazole groups and therefore expected to contribute more to ion conductivity. Since the mechanical properties are governed by the dynamics of the polystyrene backbone, the ion conductivity is decoupled from the mechanical stiffness and viscosity of the material. Such behavior can be exploited for applications such as proton exchange membranes that require both high conductivity and mechanical stability. Additionally we observed suppression of viscous flow in this material, which is ascribed to the presence of a network formed by hydrogen bonding among tetrazole groups. This property has practical utility by enabling stable membranes to be obtained without the need for chemical cross-linking.

\section{ASSOCIATED CONTENT}

\section{S Supporting Information}

Additional mechanical data. This material is available free of charge via the Internet at http://pubs.acs.org.

\section{AUTHOR INFORMATION}

\section{Notes}

The authors declare no competing financial interest.

\section{ACKNOWLEDGMENTS}

This work was supported by the Office of Naval Research. B.L.C. acknowledges the NRL Pathways Program. B.L.C. 
thanks the Pennsylvania State University and thesis advisor, Dr. Michael Hickner, for providing guidance and the opportunity to perform research at NRL.

\section{REFERENCES}

(1) Ricks-Laskoski, H. L.; Chaloux, B. L.; Deese, S. M.; Laskoski, M.; Miller, J. B.; Buckley, M. A.; Baldwin, J. W.; Hickner, M. A. Tetrazolation of Side-Chains and Anhydrous Conductivity in a Hydrophobic Polymer Macromolecules, in preparation.

(2) Granados-Focil, S.; Woudenberg, R. C.; Yavuzcetin, O.; Tuominen, M. T.; Coughlin, E. B. Water-Free Proton-Conducting Polysiloxanes: A Study on the Effect of Heterocycle Structure. Macromolecules. 2007, 40, 8708-8713.

(3) Kreuer, K. D.; Paddison, S. J.; Spohr, E.; Wilhelm, M. S. Transport in Proton Conductors for Fuel-Cell Applications: Simulations, Elementary Reactions, and Phenomenology. Chem. Rev. 2004, 104, 4637-4678.

(4) Di Noto, V.; Piga, M.; Pace, G.; Negro, E.; Lavina, S. Dielectric Relaxations and Conductivity Mechanism of Nafion: Studies Based on Broadband Dielectric Spectroscopy. ECS Trans. 2008, 16, 1183-1193.

(5) Asensio, J. A.; Sáncheza, E. M.; Gómez-Romero, P. ProtonConducting Membranes Based on Benzimidazole Polymers For HighTemperature PEM Fuel Cells. A Chemical Quest. Chem. Soc. Rev. 2010, 39, 3210-3239.

(6) Rozière, J.; Jones, D. J. Non-Fluorinated Polymer Materials for Proton Exchange Membrane Fuel Cells. Annu. Rev. Mater. Res. 2003, 33, 503-555.

(7) Pu, H.-T.; Ye, S. Preparation and Proton Conductivity of AcidDoped Poly(5-vinyltetrazole-co-acrylonitrile). React. Funct. Polym. 2006, 66, 856-862.

(8) Pu, H.; Wu, J.; Wan, D.; Chang, Z. Synthesis and Anhydrous Proton Conductivity of Poly(5-Vinyltetrazole) Prepared by Free Radical Polymerization. J. Membr. Sci. 2008, 322, 392-399.

(9) Macdonald, J. R. Theory of AC Space-Charge Polarization Effects in Photoconductors, Semiconductors, and Electrolytes. Phys. Rev. 1953, 92, 4-17.

(10) Fragiadakis, D.; Dou, S.; Colby, R. H.; Runt, J. Molecular Mobility and $\mathrm{Li}+$ Conduction in Polyester Copolymer Ionomers Based on Poly(Ethylene Oxide). J. Chem. Phys. 2009, 130, 064907.

(11) Pajkossy, T. Impedance Spectroscopy at Interfaces of Metals and Aqueous Solutions - Surface Roughness, CPE and Related Issues. Solid State Ionics 2005, 176, 1997-2003.

(12) Roland, C. M. Viscoelastic Behavior of Rubbery Materials; Oxford Univ. Press: Oxford, UK, 2011.

(13) Kudlik, A.; Benkhof, S.; Blochowicz, T.; Tschirwitz, C.; Rossler, E. The Dielectric Response of Simple Organic Glass Formers. J. Mol. Struct. 1999, 479, 201-218.

(14) Ngai, K. L.; Capaccioli, S. Relation Between the Activation Energy of the Johari-Goldstein $\beta$ Relaxation and $\mathrm{T}_{\mathrm{g}}$ of Glass Formers. Phys. Rev. E 2004, 69, 031501.

(15) Santangelo, P. G.; Roland, C. M. Molecular Weight Dependence of Fragility in Polystyrene. Macromolecules 1998, 31, 4581-4585.

(16) Fetters, L. J.; Lohse, D. J.; Milner, S. T.; Graessley, W. W. Packing Length Influence in Linear Polymer Melts on the Entanglement, Critical, and Reptation Molecular Weights. Macromolecules 1999, 32, 6847-6851.

(17) Pakula, T.; Geyler, S.; Edling, T.; Boese, D. Relaxation and Viscoelastic Properties of Complex Polymer Systems. Rheol. Acta 1996, 35, 631-640.

(18) Casalini, R.; Santangelo, P. G.; Roland, C. M. Anomalous Behavior in Blends of a Polychlorinated Biphenyl with Polystyrene Oligomer Investigated by Mechanical and Dielectric Spectroscopy. J. Phys. Chem. B 2002, 106, 11492.

(19) Casalini, R.; Santangelo, P. G.; Roland, C. M. Dynamics Of Aroclor And Its Modification By Dissolved Polystyrene. J. Chem. Phys. 2002, 117, 4585 .
(20) Santangelo, P. G.; Roland, C. M. Temperature Dependence of Mechanical and Dielectric Relaxation in cis-1,4-Polyisoprene. Macromolecules 1998, 31, 3715-3719.

(21) Buchenau, U. Mechanical and Dielectric Relaxation Spectra In Seven Highly Viscous Glass Formers. J. Non-Cryst. Solids 2007, 353, 3812-3819.

(22) Buchenau, U.; Ohl, M.; Wischnewski, A. A New Interpretation of Dielectric Data in Molecular Glass Formers. J. Chem. Phys. 2006, $124,094505$.

(23) Eicher, T.; Hauptmann, S. The Chemistry of Heterocycles: Structures, Reactions, Synthesis, and Applications; Wiley-VCH: Germany, 2003.

(24) Agmon, N. The Grotthuss Mechanism. Chem. Phys. Lett. 1995, 244, 456-462.

(25) Koike, T. Viscoelastic Behavior of Epoxy Resins Before Crosslinking. Adv. Polym. Sci. 1999, 148, 139-188.

(26) Knauth, P.; Sgreccia, E.; Donnadio, A.; Casciola, M.; Di Vona, M. L. Water Activity Coefficient and Proton Mobility in Hydrated Acidic Polymers. J. Electrochem. Soc. 2011, 158, B159-B165.

(27) Kawada, A.; McGrif, A. R.; Labes, M. M. Protonic Conductivity in Imidazole Single Crystal. J. Chem. Phys. 1970, 52, 3121-3125.

(28) Satchell, J. F.; Smith, B. Calculation of Aqueous Dissociation Constants of 1,2,4-Triazole and Tetrazole: a Comparison of Solvation Models. Phys. Chem. Chem. Phys. 2002, 4, 4314-4318. 


\title{
Supporting information for: Ion and chain mobility in a tetrazole proton-conducting polymer
}

\author{
R. Casalini, B.L. Chaloux, C.M. Roland and H.L. Ricks-Laskoski \\ Naval Research Laboratory, Chemistry Division, Code 6120, Washington DC 20375-5342
}

Mechanical measurements at high temperature.

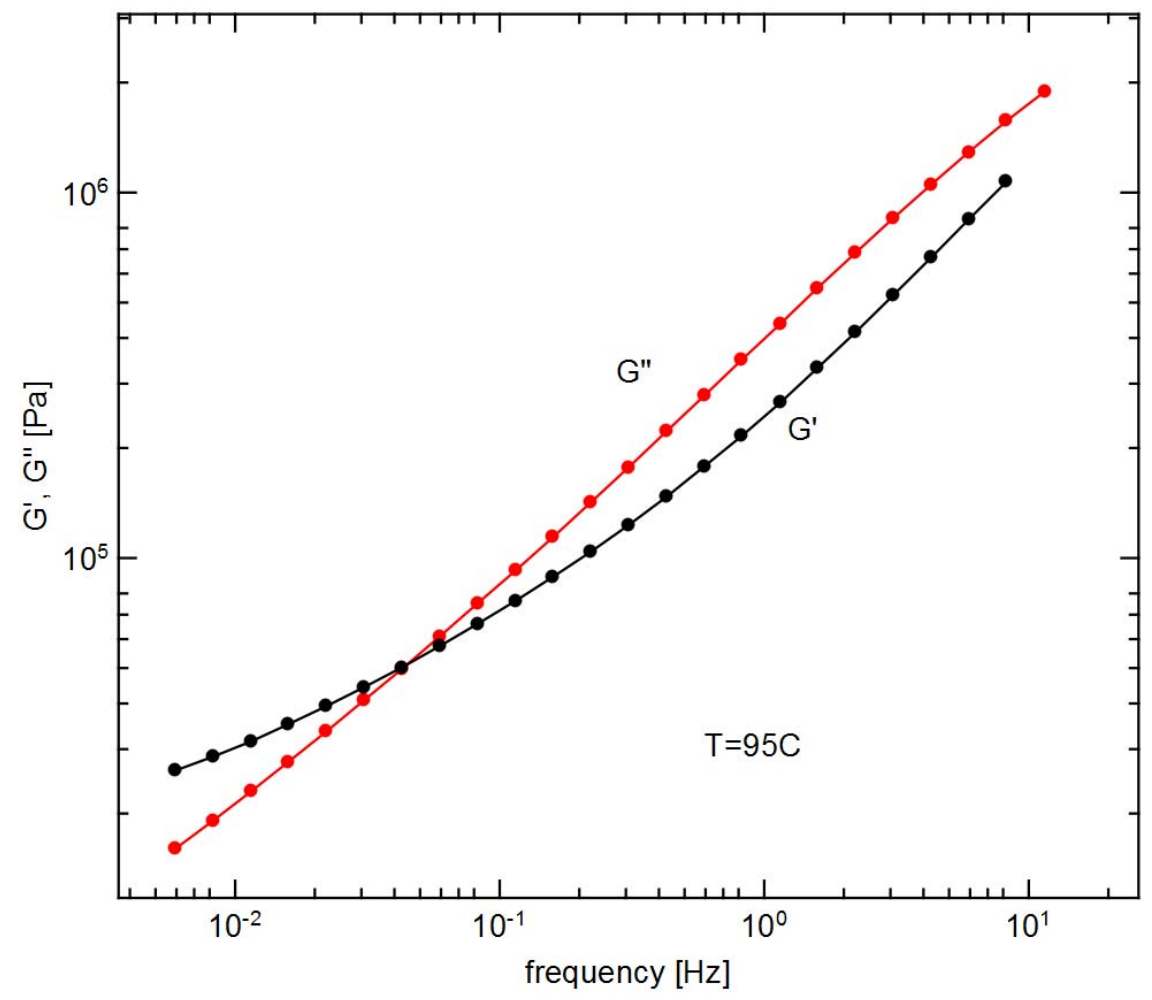

Storage and Loss moduli of PS-(Tz-Co-CN) measured at T=95C. At low frequency the slope of G' decreases 


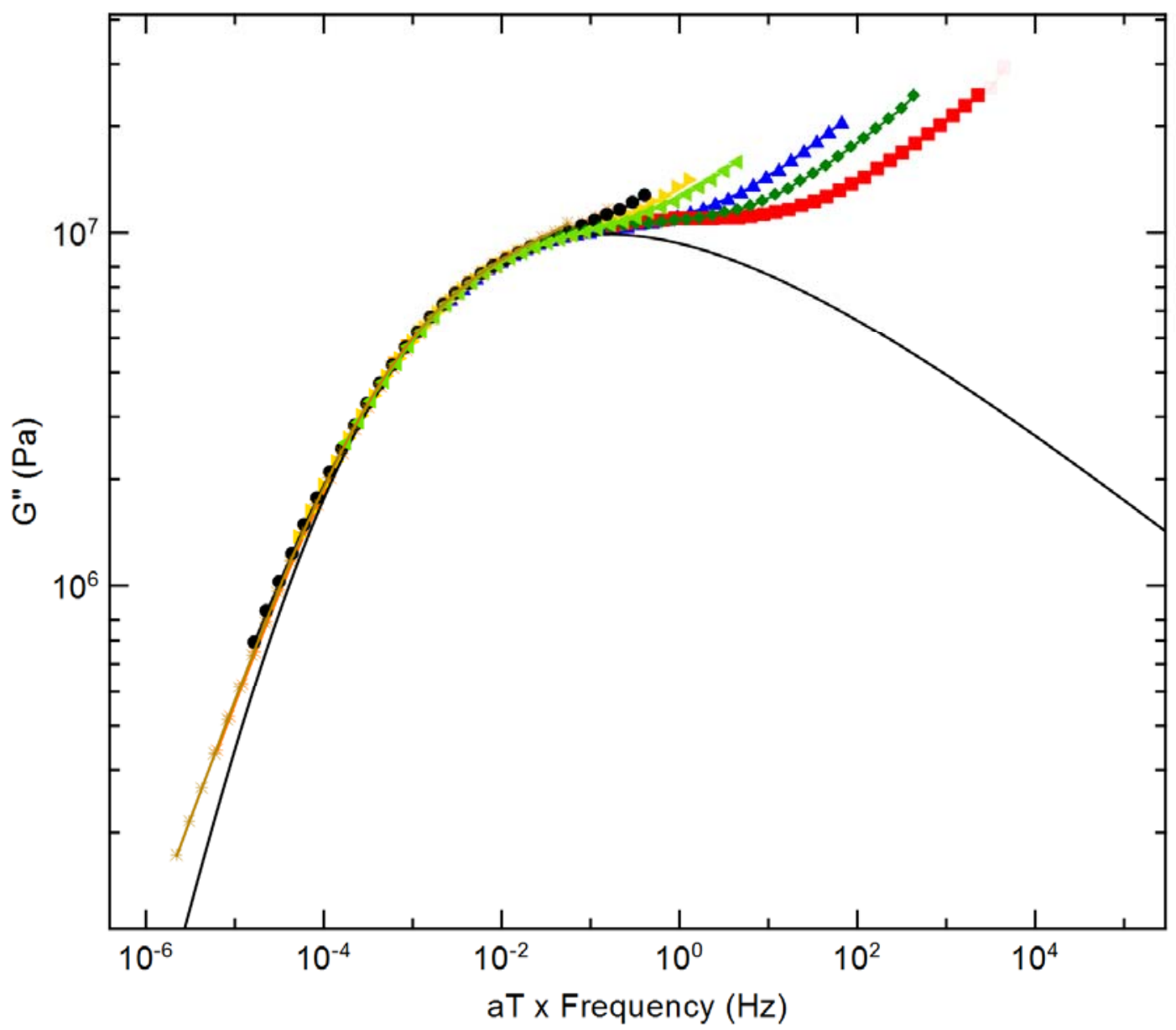

Master curve of the slow mechanical relaxation to determine the shift factor $\mathrm{a}_{\mathrm{T}}$. The solid line is the best fit to the spectra at $\mathrm{T}=56^{\circ} \mathrm{C}$ with a KWW function $\left(\log (\tau)=-0.198, \Delta \mathrm{G}=8.7 \times 10^{7} \mathrm{~Pa}, \beta_{\mathrm{kww}}=0.2\right)$. The spectra are for temperatures $\left({ }^{\circ} \mathrm{C}\right): 47,50,53,56,59,62,65,68,71$. 